\title{
Contribuições técnicas e socioculturais da prevenção quaternária para a atenção primária à saúde: caminhos e desafios
}

\author{
Technical and socio-cultural contributions of quaternary prevention for primary health care: paths \\ and challenges
}

\section{Contribuciones técnicas y socioculturales de prevención cuaternaria para la atención primaria de salud: caminos y desafíos}

\author{
Fernanda Beatriz Melo Maciel ${ }^{\circledR}$, Hebert Luan Pereira Campos dos Santos $^{1 \oplus}$, Nilia Maria de Brito Lima Prado ${ }^{\circledR}$ \\ ${ }^{1}$ Universidade Federal da Bahia, Instituto Multidisciplinar em Saúde, Vitória da Conquista, Salvador, BA, Brasil.
}

\section{Resumo}

Introdução: No contexto do Sistema Único de Saúde, o conceito da prevenção quaternária adentra timidamente os níveis de atenção à saúde, no entanto, sofre expansão significativa no âmbito da atenção primária à saúde. Objetivo: Identificar por meio da sistematização de evidências científicas, as contribuições técnicas e socioculturais da prevenção quaternária no âmbito da atenção primária à saúde no Brasil. Métodos: Trata-se de uma revisão integrativa de estudos presentes nas bases de dados científicas da Scientific Electronic Library Online, Biblioteca Virtual em Saúde, biblioteca virtual da Comissão de Aperfeiçoamento de Pessoal do Nível Superior e MEDLINE via PubMed com a utilização dos descritores "prevenção quaternária" e "atenção primária à saúde", em inglês e português. Resultados: $O$ corpus de análise foi composto por 22 artigos, sendo que a produção científica sobre o tema se deu de forma mais intensa a partir do ano de 2015 e, em sua maioria, possuíam como abordagem metodológica ensaios teóricos. Dentre as contribuições técnicas destacaram-se a introdução do ensino da prevenção quaternária de modo continuado aos graduandos e profissionais; a construção de protocolos e documentos de amparo profissional; a utilização de modelos explicativos dinâmicos na socialização do quadro clínico; a conduta profissional com os usuários e as contribuições socioculturais envolvendo mudanças na percepção profissional e comunitária sobre o fenômeno saúde-doença, assim como o incentivo a práticas de desmedicalização sociocultural em relação à dor, incapacidade, desconforto, envelhecimento, nascimento e morte. Conclusão: Apesar do reconhecimento das potencialidades da prevenção quaternária, faz-se necessário fortalecer estratégias que possibilitem o desenvolvimento de políticas públicas para fomentar e gerenciar alianças estratégicas com tomadores de decisão, profissionais de saúde e cidadãos, para fomentar a redução de diagnósticos e tratamentos excessivos, contribuindo com a qualidade do cuidado.

Palavras-chave: Prevenção Quaternária; Atenção Primária à Saúde; Sistema Único de Saúde; Medicina de Família e Comunidade; Sobremedicalização.

Como citar: Maciel FBM, Santos HLPC, Prado NMBL. Contribuições técnicas e socioculturais da prevenção quaternária para a atenção primária à saúde: caminhos e desafios. Rev Bras Med Fam Comunidade. 2020;15(42):2571. https://doi.org/10.5712/rbmfc15(42)2571

Autor correspondente:

Fernanda Beatriz Melo Maciel.

E-mail: fernandamlmcl728@gmail.com

Fonte de financiamento:

declaram não haver.

Parecer CEP:

não se aplica.

Procedência:

não encomendado.

Avaliação por pares:

externa.

Recebido em: 15/06/2020.

Aprovado em: 26/10/2020. 


\begin{abstract}
Introduction: In the context of the Unified Health System, the concept of quaternary prevention shyly enters the levels of health care, however, undergoes significant expansion in the scope of primary health care. Objective: To identify, through the systematization of scientific evidence, the technical and socio-cultural contributions of quaternary prevention within the scope of primary health care in Brazil. Methods: This is an integrative review of studies present in the scientific databases of the Scientific Electronic Library Online, Regional Portal of the Virtual Health Library of the Latin American and Caribbean Center on Health Sciences Information of the Pan American Health Organization, virtual library of the Higher Education Personnel Improvement Commission, and MEDLINE through PubMed using the descriptors "quaternary prevention" and "primary health care", in English and Portuguese. Results: The corpus of analysis consisted of 22 articles, and the scientific production on the topic took place more intensively from the year 2015 and, for the most part, had theoretical essays as methodological approach. Among the technical contributions, we highlight the introduction of teaching on quaternary prevention in a continuous way to undergraduates and professionals; the construction of protocols and documents of professional support; the use of dynamic explanatory models in the socialization of the clinical picture and professional conduct with users and socio-cultural contributions involve changes in the professional and community perception about the phenomenon of illness and health conception, as well as the incentive to practices of socio-cultural demedicalization in relation to pain, disability, discomfort, aging, birth, and death. Conclusion: Despite the recognition of the potential of quaternary prevention, it is necessary to strengthen strategies that enable the development of public policies to foster and manage strategic alliances with decision makers, health professionals and citizens, to promote the reduction of excessive diagnoses and treatments, contributing to the quality of care.
\end{abstract}

Keywords: Quaternary Prevention; Primary Health Care; Unified Health System; Family and Community Medicine; Medical Overuse.

\title{
Resumen
}

Introducción: En el contexto del Sistema Único de Salud, el concepto de prevención cuaternaria entra tímidamente en los niveles de atención de salud, sin embargo, experimenta una expansión significativa en el alcance de la Atención Primaria de Salud. Objetivo: Identificar, a través de la sistematización de evidencia científica, las contribuciones técnicas y socioculturales de la prevención cuaternaria en el ámbito de la Atención Primaria de Salud en Brasil. Métodos: Esta es una revisión integradora de estudios presentes en las bases de datos científicas de la Biblioteca Electrónica Científica en línea, Portal Regional de la Biblioteca Virtual en Salud del Centro Latinoamericano y del Caribe de Información en Ciencias de la Salud de la Organización Panamericana de la Salud, biblioteca virtual de la Comisión de Mejoramiento del Personal de Educación Superior y MEDLINE a través de PubMed utilizando los descriptores de prevención cuaternaria y atención primaria de salud, en inglés y portugués. Resultados: El corpus de análisis estuvo conformado por 22 artículos, siendo la producción científica sobre el tema más intensiva desde 2015 y, en su mayor parte, tuvo ensayos teóricos como abordaje metodológico. Entre los aportes técnicos, destacamos la implantación de la docencia en prevención cuaternaria de forma continua a estudiantes de pregrado y profesionales; construcción de protocolos y documentos de apoyo profesional, uso de modelos explicativos dinámicos en la socialización del cuadro clínico y conducta profesional con los usuarios y los aportes socioculturales implican cambios en la percepción profesional y comunitaria sobre el fenómeno de la enfermedad y la concepción de la salud, así como el incentivo a prácticas de desmedicalización sociocultural en relación al dolor, discapacidad, malestar, envejecimiento, nacimiento y muerte. Conclusión: A pesar del reconocimiento del potencial de la prevención cuaternaria, es necesario fortalecer estrategias que permitan el desarrollo de políticas públicas para fomentar y gestionar alianzas estratégicas con los tomadores de decisiones, profesionales de la salud y ciudadanos, para promover la reducción de diagnósticos y tratamientos excesivos, contribuyendo a la calidad de la atención.

Palabras clave: Prevención Cuaternaria; Primeros Auxilios; Sistema Único de Salud; Medicina Familiar y Comunitaria; Uso Excesivo de los Servicios de Salud.

\section{INTRODUÇÃO}

O lastro teórico fundamental acerca da prevenção quaternária é considerado recente na área médica apesar de, nas últimas décadas, diversos autores terem ampliado o debate contemplando novos olhares sobre a prevenção quaternária na rede de atenção à saúde, incluindo discussões que permeiam a prática médica-sanitarista baseada em evidências clínicos-científicas em detrimento à sobremedicalização, sobrecarga de informação e outros. ${ }^{1}$

A preocupação acerca das práticas preventivas acentuou-se na primeira metade do século $\mathrm{XX}, \mathrm{com}$ o desenvolvimento da medicina preventiva, a qual revela-se como movimento em prol da construção de uma nova atitude médica que seja capaz de descentralizar posturas curativistas. ${ }^{2,3}$

Em 2000, Jamoulle $e^{4}$ evidenciou a pertinência de aplicar os princípios da prevenção quaternária dentro de todos os níveis de prevenção apresentados por Leavell e Clark, em 1976. ${ }^{1}$ A proposta, nesse contexto, 
tinha por finalidade identificar pessoas ou populações em "risco de supermedicalização, para protegê-los de intervenções médicas invasivas e sugerir-lhes procedimentos que do ponto de vista ético e científico fossem aceitáveis." (p. 2). ${ }^{5}$

Nessa direção, o Comitê Internacional de Classificação da WONCA(Organização Mundial de Colégios Nacionais, Academias e Associações Acadêmicas de Médicos de Família) incorporou esse conceito ao seu dicionário preservando os ideais cunhados por Jamoulle (2000). ${ }^{4}$ Mais recentemente, Martins el at. (2018), ${ }^{6}$ apontaram que o modelo conceitual proposto restringe o seu campo de ação às situações em que o paciente apresenta constantes queixas e um determinado significado ao processo de adoecimento, sem necessariamente evidenciar sinais, sintomas e alterações em exames físicos e complementares que auxiliariam na identificação da doença referida, demonstrando assim que o médico deveria se abster a realizar testes invasivos potencialmente prejudiciais apenas a esses pacientes. Nesse sentido, os autores indicam a necessidade de uma conceituação que torne todos os indivíduos e populações elegíveis à prevenção quaternária. ${ }^{6}$ Nessa lógica, Brodersen et al. (2014), ${ }^{7}$ definiram a prevenção quaternária como ações tomadas para proteger indivíduos de intervenções médicas que são provavelmente mais maléficas do que benéficas, esclarecendo assim que a prevenção quaternária deve estar presente cotidianamente na prática médica para guiar cada intervenção que eles sugerirem a um paciente.

No contexto brasileiro, esse conceito adentra timidamente nos níveis de atenção à saúde, mas sofre expansão, principalmente, no âmbito da atenção primária à saúde (APS). Isso porque a APS constitui o nível de atenção que utiliza no processo de cuidado tecnologias de relações pautadas na produção de vínculo, autonomização, acolhimento e um menor aporte de tecnologias duras, de modo a reposicionar a prática clínica e reduzir as iatrogenias presentes no processo de trabalho da equipe de saúde, se aproximando com o preconizado pela prevenção quaternária. 3,8

Nesse sentido, o presente estudo objetivou identificar, através da sistematização de evidências científicas, as contribuições técnicas e socioculturais da prevenção quaternária no âmbito da APS no Brasil.

A construção deste artigo justifica-se, portanto, ao reconhecer que o entendimento das contribuições da prevenção quaternária no contexto da APS pode reduzir a adoção de práticas iatrogênicas, intervenções médicas desnecessárias, sobremedicalização, entre outras práticas que vão de encontro aos princípios da prevenção quaternária e impactam diretamente a saúde individual, coletiva e a efetividade do sistema de saúde.

\section{MÉTODOS}

Trata-se de uma revisão integrativa sobre a prevenção quaternária no contexto da APS no Brasil que buscou responder às seguintes questões de investigação: em que medida a prevenção quaternária tem sido incluída nas políticas e práticas de saúde implementadas no âmbito da APS? Quais as contribuições técnicas e socioculturais desta para o desenvolvimento das ações na APS?

Revisores independentes realizaram a busca de artigos nos meses de abril e agosto de 2020, nas bases de dados científicas da Scientific Electronic Library Online (SciELO); Portal Regional da Biblioteca Virtual em Saúde (BVS), Biblioteca Virtual da Comissão de Aperfeiçoamento de Pessoal do Nível Superior (CAPES) e MEDLINE via PubMed, sendo as estratégias de busca utilizadas compostas pelos descritores, consultados na plataforma do Descritores em Ciências da Saúde (DeSC) e no MeSh (vocabulário controlado 
da NLM), associados ao operador booleano AND: "prevenção quaternária AND atenção primária à saúde" e "quaternary prevention AND primary health care".

Adotou-se como critério de inclusão estudos que abordavam a temática proposta, publicados em português e inglês, disponíveis na íntegra. Foram excluídos artigos duplicados, em outros idiomas e que não abordavam a temática de prevenção quaternária ou cuja abordagem não contribuísse para o conhecimento da área pretendida. Não houve exclusão por delimitação temporal e abordagem metodológica.

Para fins de análise, os artigos foram organizados em um banco de dados no software Microsoft Exce $®$, considerando as seguintes variáveis: ano da publicação, título, nome dos autores, periódico, desenho/estratégia de estudo e principais achados, sendo esses dois últimos definidos inicialmente a partir do resumo do artigo e confirmados pela leitura do artigo na íntegra. A sistematização envolveu as etapas de identificação, fichamento, análise e interpretação dos estudos selecionados.

Foram identificadas inicialmente 195 publicações, 49 dessas foram excluídas após verificada a duplicação nas bases de dados, 73 após aplicação dos filtros e 51 por não discutirem a temática central, restando 22 artigos para compor o corpus deste estudo. Do total, 4 artigos foram identificados na BVS, 10 na CAPES, 6 na SciELO e 2 na PubMed (Figura 1). Após esta etapa, foi realizada a leitura crítica dos 22 artigos selecionados. A partir das evidências oferecidas pelo corpus de análise realizou-se o cálculo de frequência simples, a fim de caracterizar os artigos encontrados.

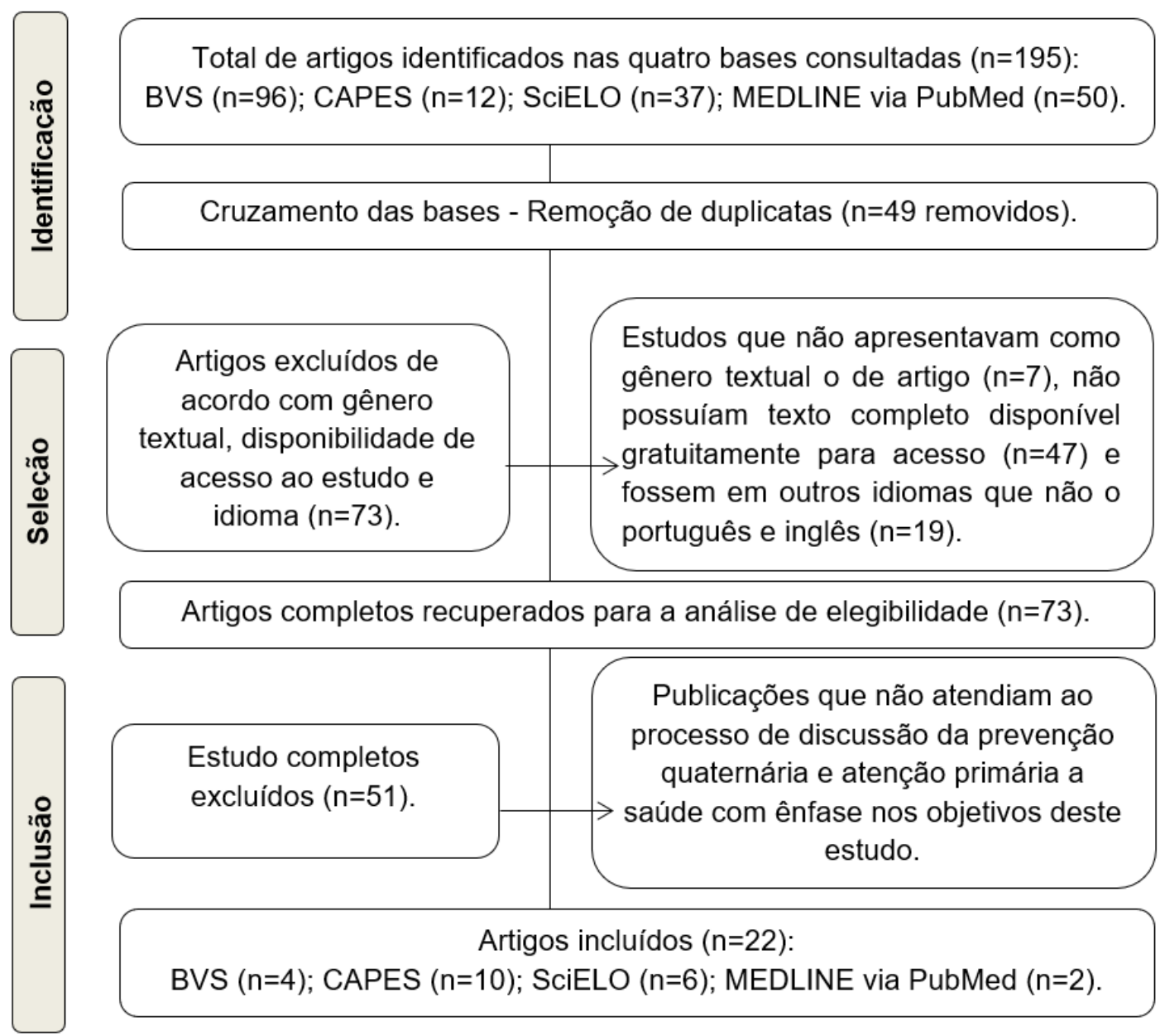

Figura 1. Fluxograma da sistematização do levantamento bibliográfico. 


\section{RESULTADOS}

Os artigos analisados foram publicados, majoritariamente, a partir do ano de 2015 (86,5\%), veiculados em diferentes periódicos, entretanto, constatou-se um maior número de publicações sobre a temática na Revista Brasileira de Medicina de Família e Comunidade (37\%). No que se refere ao tipo de abordagem da pesquisa, tratavam-se predominantemente de ensaios teóricos $(68,5 \%)$ (Quadro 1).

Quadro 1. Caracterização dos artigos selecionados, segundo ano de produção, periódico publicado e abordagem metodológica adotada.

\begin{tabular}{|c|c|c|}
\hline Ano de publicação & $\mathbf{N}$ & $\%$ \\
\hline 2009 & 1 & 4,5 \\
\hline 2012 & 2 & 9 \\
\hline 2016 & 2 & 9 \\
\hline 2017 & 3 & 14 \\
\hline 2019 & 5 & 23 \\
\hline 2020 & 1 & 4,5 \\
\hline TOTAL & 22 & 100 \\
\hline Periódico & $\mathbf{N}$ & $\%$ \\
\hline Saúde e Sociedade & 1 & 4,5 \\
\hline Revista Eletrônica Gestão \& Saúde & 1 & 4,5 \\
\hline Trabalho, educação e saúde & 1 & 4,5 \\
\hline Acta Méd. & 1 & 4,5 \\
\hline Revista Enfermagem UFPE & 1 & 4,5 \\
\hline O Mundo da Saúde & 1 & 4,5 \\
\hline Revista de Saúde Pública & 1 & 4,5 \\
\hline BMC Family Practice & 1 & 4,5 \\
\hline Revisão narrativa & 1 & 4,5 \\
\hline Revisão sistemática & 1 & 4,5 \\
\hline Revisão integrativa & 1 & 4,5 \\
\hline Estudo qualitativo & 2 & 9 \\
\hline Estudo quantitativo & 1 & 4,5 \\
\hline TOTAL & 22 & 100 \\
\hline
\end{tabular}

Fonte: Elaboração própria. 
A partir da análise do corpus bibliográfico selecionado, verificou-se que os 22 estudos selecionados ${ }^{3,8-29}$

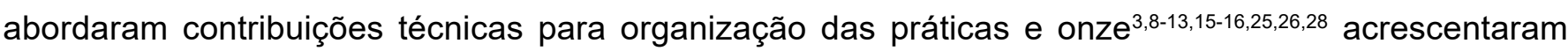
contribuições socioculturais. Partindo disso, elaborou-se um quadro (Quadro 2) buscando sintetizar contribuições possíveis à execução, gestão e formação de sistemas de saúde e recursos humanos para a prática da prevenção quaternária na APS.

Quadro 2. Quadro síntese das contribuições da prevenção quaternária para o desenvolvimento das ações na APS.

\begin{tabular}{|c|}
\hline Contribuições \\
\hline Incluir na educação médica o ensino teórico e prático acerca da prevenção quaternária. 5,18,23,25,28 \\
\hline $\begin{array}{l}\text { Construir protocolos interpares e documentos de orientação gerencial que norteiem a verificação e desencorajem o uso médico excessivo } \\
\text { aplicando o cuidado em saúde baseado em evidências. }{ }^{9,12,25,29}\end{array}$ \\
\hline Utilizar modelos explicativos dinâmicos na socialização com os usuários para elucidação do quadro clínico e condutas médicas. ${ }^{13}$ \\
\hline Fomentar pesquisas visando ampliar as evidências científicas sobre sobremedicalização e outras práticas médicas excessivas. 11,22,24,25,28 $^{-18}$ \\
\hline Realizar um cuidado em saúde centrado na pessoa. ${ }^{3,9,10,11,20}$ \\
\hline $\begin{array}{l}\text { Centrar o cuidado na atenção primária à saúde, com longitudinalidade, aplicando a "demora permitida" e conduzindo a investigação } \\
\text { diagnóstica de forma gradual com foco na anamnese e no exame físico. }{ }^{3,8-12,25,26}\end{array}$ \\
\hline Construir relações de trabalho em saúde pautada na interprofissionalidade e na prática colaborativa. . $3,9,10,23^{2}$ \\
\hline Incluir o paciente na construção dos projetos terapêuticos através da comunicação dialógica. ${ }^{3,9,10,20}$ \\
\hline $\begin{array}{l}\text { Considerar durante a prática clínica a experiência profissional, clínica do paciente, preferência do paciente e familiares, norteado pelo } \\
\text { questionamento ético e reflexivo. }{ }^{2,16,23,24}\end{array}$ \\
\hline Promover campanhas sobre sobremedicalização e intervenções médicas excessivas para comunidades e profissionais. ${ }^{11,26}$ \\
\hline Envolver diferentes profissionais de saúde na discussão e no processo de definição de causas e soluções do uso médico excessivo. ${ }^{8-12,25,29}$ \\
\hline $\begin{array}{l}\text { Incentivar práticas de desmedicalização sociocultural em relação à dor, incapacidade, desconforto, envelhecimento, nascimento e } \\
\text { morte. }{ }^{2,8,9,13}\end{array}$ \\
\hline udanças na percepção profissional e comunitária sobre o fenômeno do adoecimento e conce \\
\hline
\end{tabular}

Fonte: Elaboração própria a partir da análise do corpus bibliográfico selecionado.

\section{DISCUSSÃO}

A sistematização das discussões apresentadas pelos autores ${ }^{3,8-29}$ permite compreender, de forma mais abrangente, as contribuições da prevenção quaternária para organização das práticas, como uma ação preventiva direcionada ao agir profissional clínico-sanitário com ênfase no desenvolvimento de ações e desdobramentos práticos e/ou técnicos guiados pela resistência ética, atitudinal, epistemológica e política aos excessos de iatrogenia, preventivismo e medicalização nas práticas profissionais e institucionais, desdobrando-se também numa mudança de perspectiva da sociedade.

Gross et al. (2016) ${ }^{18}$ apontam que a inclusão da prevenção quaternária pode ser comprometida ante à falta de capacitação profissional e a fragilidade das políticas de educação permanente. Nesse sentido, a consolidação dessa prevenção na APS perpassa, inicialmente, pelo entendimento por parte dos profissionais sobre o que é a prevenção quaternária, quais seus objetivos e finalidades.

Para o alcance desse entendimento, os artigos analisados ${ }^{5,23,25,28}$ propõem a inclusão da prevenção quaternária nos currículos em saúde vislumbrando uma mudança de conduta dos profissionais frente a influência da cascata diagnóstica e preventiva desnecessária, dos modismos e da pressão exercida pela corporação profissional-tecnológico-farmacêutica. 
O caminho proposto, enquanto contribuição técnica e sociocultural, inclui ao processo formativo dos futuros profissionais de saúde e dos profissionais já existentes a educação permanente para uma mudança de postura na construção do cuidado em saúde. ${ }^{18}$ Salienta-se, contudo, que a sua consolidação a nível de serviço perpassa pela sua integralidade no processo formativo dos novos profissionais, de forma transversal, com um quadro teórico-prático sólido e consistente, desde os semestres iniciais. ${ }^{28}$ Conjectura-se, nesse sentido, a possibilidade de maior compreensão e distinção entre os conceitos e finalidades dos níveis de prevenção, uma vez que, na prática, nota-se que esses não são identificados em suas peculiaridades e a prevenção quaternária, sobretudo, não se faz suficientemente esclarecida. ${ }^{26}$

Almenas et al. (2018) ${ }^{30}$ sugerem ampliar os mecanismos educacionais, abrangendo a educação e as orientações em saúde para população e gestores de instituições de ensino e serviços de saúde, sobretudo, para que possam encontrar informação qualificada e capacitação para o ensino das práticas da prevenção quaternária. Nesse ponto, deve-se pensar que a promoção de campanhas, fóruns, eventos científicos e outras formas de divulgação para profissionais e comunidades podem ser uma das estratégias a serem adotadas para difusão do conhecimento a respeito da prevenção quaternária. ${ }^{11,26}$

De forma prática, apesar da proposição para identificar pessoas e populações em risco de supermedicalização e sugerir-lhes procedimentos cientificamente e eticamente aceitáveis, observa-se a carência de bases diretivas através de normas, protocolos, diretrizes e outros documentos que deliberem a abrangência da prevenção quaternária para profissionais.

Nessa direção, a construção de protocolos interpares e documentos de orientação são apontados como alguns dos instrumentos que podem ser utilizados para garantir que o profissional se atualize sobre o cuidado em saúde baseado em evidências, evitando a utilização de práticas que careçam de evidências científicas para apoiá-las. ${ }^{7-12,25,29}$ Soma-se a isso a ampliação de pesquisas que envolvam diretamente as premissas da prevenção quaternária nas práticas de saúde, buscando compreender também o que a comunidade entende por sobremedicalização e uso excessivo da medicina. ${ }^{11}$ Supõe-se que a existência desses documentos estabeleçam um embasamento, até mesmo do ponto de vista jurídico-legal, ao registro das decisões de não seguir determinada conduta visando à prática da prevenção quaternária sem que esse profissional seja compelido pela percepção da negligência médica. ${ }^{22,24,25,28}$

No contexto da APS, Tesser e Norman (2019) ${ }^{13}$ atestam que a detecção de indivíduos e grupos em risco de tratamento excessivo requer uma interpretação dinâmica das situações clínicas, para assim checar cuidadosamente os sinais de alarme apresentados pelo paciente, ante a aplicação demasiada de intervenções e medicamentos. ${ }^{23}$ É consensual entre os estudos ${ }^{3,8-12,25,26}$ que o acompanhamento longitudinal, centrado na APS, acrescido de uma postura profissional que explore a doença e experiência do adoecer são necessários para compreender a pessoa, o processo de adoecimento e negociar um plano comum de manejo.

É circunstancial, ainda, que o profissional reconheça, frente ao risco de sobremedicalização, a vulnerabilidade dos indivíduos sem sintomas que frequentemente procuram um médico; dos pacientes em situação paliativa que recebem o máximo de investigações e tratamento; pacientes com diagnóstico psicossomático que prioriza a saúde somática em vez da psicoterapia; pacientes idosos e multimórbidos que correm risco de polifarmacoterapia. ${ }^{9,22}$ 
Ainda enquanto contribuição técnica, buscando auxiliar o processo de trabalho dos profissionais de saúde, Harzheim et al. (2019) ${ }^{14}$ propõem a integração cautelosa da telessaúde, a qual poderá auxiliar na racionalização da ocorrência de sobremedicalização e de iatrogenias médico-sanitárias por meio da melhoria do acesso. ${ }^{14}$ Outros estudos ${ }^{30,31}$ colaboram com essa perspectiva ao apontar a telemedicina como instrumento capaz de melhorar a qualidade da atenção à saúde, reduzir o tempo gasto entre o diagnóstico e a terapia, assim como promover a racionalização medicamentosa, requerendo, entretanto, constante capacitação profissional.

Embora seja amplamente difundido que a prevenção quaternária está centrada e restrita ao campo da medicina, especialmente, da medicina de família, ${ }^{8,9,28,29,33-36}$, fator que corrobora para a dualidade na introdução das suas práticas na APS e nos outros níveis de atenção, as premissas defendidas por esse tipo de prevenção, ainda que possuam centralidade para o profissional médico e maior capilaridade na APS, são premissas que devem ser adotadas por todos os profissionais de saúde e em todos os níveis de atenção. ${ }^{26}$

A prática de todo profissional de saúde deve ser guiada, desse modo, pelo cuidado em saúde baseado em evidências, o qual permitirá embasar, técnica e eticamente, as decisões clínicas 3,8,9,10,11,20,21,22,37. Norman et al. (2017), ${ }^{21}$ nesse sentido, destaca a importância do manifesto de medicina baseada em evidências para melhor assistência à saúde, o qual busca promover uma visão mais abrangente e holística da relação médico-paciente e reduzir a adoção de práticas questionáveis de pesquisa, vieses e conflitos de interesses. ${ }^{21,24-27}$ Ressalta-se também que a prevenção quaternária propõe a realização de um cuidado em saúde centrado na pessoa, o que envolve diretamente a aliança entre experiência profissional, clínica do paciente, preferências do indivíduo e de seus familiares e, sobretudo, uma comunicação dialógica que inclua o indivíduo na construção do projeto terapêutico subsidiado por evidências científicas. 3,9,10,11,19

Em síntese, os estudos ${ }^{38,39}$ apontaram avanços importantes na discussão da própria prática profissional, dentre eles a relativização do saber e a construção da prática clínica dialógica, mas reforçam que ainda é preciso avançar na implementação de medidas que assegurem a informação atualizada e qualificada, para subsidiar e orientar as condutas profissionais, sem restringir a autonomia frente à verificação da necessidade ou não de intervenções.

Tesser (2017) $)^{12}$ e Modesto (2019) ${ }^{15}$ reconhecem que a emergência das práticas em saúde norteadas pela prevenção quaternária colide com questões do paradigma biomédico que institucionalizam-se, como força social-simbólica, através do conjunto de saberes e práticas socio-históricas que servem de referência para profissionais de saúde.

No âmbito dos sistemas de saúde isso perpassa pela avaliação e reação crítica dos profissionais de saúde, gestores e acadêmicos sobre sua postura e práxis profissional, de forma operacional, questionando seus limites técnicos e éticos, e reconhecendo as influências que incidem sobre as suas decisões e condutas preventivas. ${ }^{3,8-13,20,27}$ Esse questionamento ético incentivado, fortemente, pela prevenção quaternária, baseiase na ideia de que as práticas em saúde requerem a proteção dos interesses comerciais, o marketing de doenças e das crenças voltadas à necessidade excessiva de exames e tratamentos preventivos. ${ }^{3,8,9,13,34,35}$

Assumir a postura de resistência à pressão do marketing da indústria farmacêutica, tecnológica, profissional e populacional 3,8,9,13,34,35 requer uma série de aspectos que envolve a principal contribuição sociocultural proposta pela prevenção quaternária: incentivar práticas de desmedicalização socioculturais, 
tanto da sociedade como dos próprios profissionais. Nessa perspectiva, a percepção acerca do fenômeno do adoecimento e a concepção de saúde corroboram para a busca e alcance de maneiras de reestruturação das práticas de cuidado que se desvincule da sobrecarga diagnóstica e preventiva. 3,8-10,12, 20

Para que essa contribuição sociocultural proposta pela prevenção quaternária se cumpra faz-se necessário resgatar as dimensões étnicas, psicossociais, econômicas e comportamentais que envolvem o processo de adoecimento e a medicalização da contemporaneidade. ${ }^{3}$ Do mesmo modo, refletir como esses fatores auxiliam ou não na adesão e aceitação desse nível de prevenção em nível comunitário e individual é um dos caminhos. ${ }^{7,8,16}$ Essas ações favorecerão para que as reflexões adentrem às comunidades, produzindo um sentimento de desencanto com as práticas médicas sobremedicalizadas e iatrogênicas. ${ }^{3}$

Nesse passo Modesto (2019) $)^{15}$ e Tesser $(2019)^{16}$ enfatizam a importância do engajamento de grupos, a seguir pelo exemplo de médicas(os) de família e comunidade, as(os) quais frente a identificação de riscos e limitações das ações preventivas promovem uma reflexão acerca da necessidade de ressignificação cultural do cuidado com ênfase na valorização da escuta e elaboração de planos compartilhados de cuidado, tensionando a relevância do saber popular, em nível individual, familiar e comunitário. ${ }^{12,16,23,24}$

O processo de mudança cultural dos profissionais, para além das contribuições já propostas, envolve também a construção de práticas de trabalho interprofissionais pautadas na prática colaborativa, diálogo,

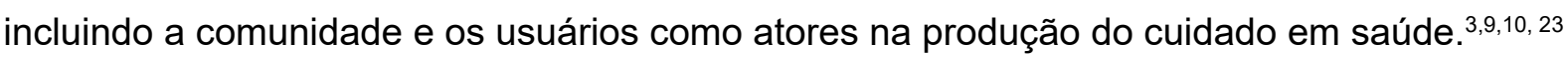

Durante o encontro clínico, o profissional também assume um papel de influenciador da mudança de postura e visão sobre o processo de adoecimento e saúde para os indivíduos que atendem. Alguns autores $^{3,9,10,20}$ consideram que a comunicação dialógica, ${ }^{38,39}$ o uso prudente das palavras, $\square$ a adoção de modelos explicativos dinâmicos na socialização com os usuários das interpretações profissionais (diagnósticos), a singularização de terapêuticas e da distinção entre cuidado ao adoecimento presente e futuro (prevenção)." (p. 3), ${ }^{7}$ são primordiais na efetivação da prática da prevenção quaternária.

Como observado, existem importantes contribuições propostas pela prevenção quaternária para a APS, para os profissionais de saúde e a comunidade, no entanto, destacam-se também grandes desafios para efetivação desse tipo de prevenção. Não se pode perder de vista o desafio ético e moral envolvido na consolidação da prevenção quaternária nos serviços e nas práticas de saúde, pois enquanto não existirem políticas e ações concretas para a implementação das premissas desse tipo de prevenção a prática permanece à mercê das atitudes e condutas profissionais. Há também um desafio político que envolve a construção de políticas públicas, as quais permitam ampliar a sua aplicabilidade e acessibilidade junto aos serviços e sistemas de saúde e também às políticas educacionais.

Deve-se destacar que o presente estudo possui algumas limitações: as contribuições aqui levantadas partiram da interpretação dos pesquisadores que construíram esse estudo sobre o corpus bibliográfico analisado e a quantidade incipiente de artigos que respondessem à pergunta de investigação que balizou a construção desse estudo. A temática da prevenção quaternária encontra-se em crescimento e a maioria dos artigos que compuseram o corpus de análise possuíam como abordagem metodológica ensaio teórico. Essas limitações poderão servir de oportunidade para o desenvolvimento de estudos e novas investigações que apresentem a incorporação e os relatos dessas premissas de modo mais prático no cotidiano da APS no SUS. 
Sendo assim, percebe-se que as contribuições socioculturais e técnicas para consolidação e ampliação da prevenção quaternária na APS e nos outros níveis de atenção abrangem a necessidade do fortalecimento de um perfil profissional que diversifique o seu olhar sobre o processo doença-saúde-cuidado; o reconhecimento político, econômico e cultural da prevenção quaternária, seja no âmbito das forças que regem as diretrizes de saúde quanto as educacionais, de modo a priorizar a oferta do melhor cuidado, valorizar as desigualdades culturais na percepção da saúde, doença e as intervenções direcionadas a cada indivíduo, família e comunidade.

\section{CONSIDERAÇÕES FINAIS}

Esta revisão possibilitou reconhecer as possíveis contribuições da prevenção quaternária no âmbito da APS no Brasil. O escopo de análise das publicações científicas desse artigo evidencia ausência de diretrizes e normativas tanto para guiar a prática clínica pautada na prevenção quaternária, quanto para subsidiar a sua operacionalização. Ademais, os estudos analisados demonstram a necessidade do desenvolvimento de pesquisas com novas abordagens metodológicas relacionadas à temática.

A prevenção quaternária na APS ainda enfrenta limitações importantes para sua execução, mesmo possuindo uma trajetória positiva no que diz respeito às contribuições técnicas e socioculturais para a prática clínica e para os serviços de saúde. Nota-se a carência de estudos que aprofundem a temática em nível nacional, de modo a exemplificar e elucidar as realidades e as medidas tomadas pelos profissionais para a sua efetivação, assim como os seus impactos frente ao cuidado em saúde. Cabe destacar que o tema ainda se coloca como uma discussão recente e pouco aprofundada pelos estudos no contexto da APS brasileira.

Para garantia de uma plena efetivação das contribuições da prevenção quaternária na APS, elencouse algumas recomendações com vistas a auxiliar nesse processo:

- Reformulação dos currículos dos cursos de saúde com vistas a inclusão da temática da prevenção quaternária, princípios da interprofissionalidade e prática colaborativa.

- Inclusão da prevenção quaternária nas políticas de saúde e nas agendas de pesquisa do país.

- Elaboração de eventos científicos com temática voltada diretamente para prevenção quaternária na APS e em outros níveis de atenção.

- Engajamento da comunidade nas decisões e elaborações de políticas públicas acerca da saúde com vistas à atenuação da medicalização excessiva da saúde.

Por fim, reconhece-se que embora as discussões sobre a prevenção quaternária sejam articuladas pelos profissionais e gestores da APS, para viabilizar a ampliação do debate sobre práticas pautadas nesta prevenção se faz extremamente necessárias mudanças de orientações normativas que possam ter impactos na garantia dos atributos essenciais da APS, especialmente a integralidade.

\section{Contribuições dos autores}

Todos os autores contribuíram substancialmente na concepção e no planejamento do estudo, na obtenção, análise e/ou interpretação dos dados, assim como na redação e/ou revisão crítica e aprovaram a versão final a ser publicada. 


\title{
Declaração de conflito de interesses
}

\author{
Nada a declarar.
}

\section{REFERÊNCIAS}

1. Leavell H, Clark EG. Medicina Preventiva. São Paulo: McGraw-Hill, 1976.

2. Jamoulle M, Gomes LF. Prevenção quaternária e limites em medicina. Rev Bras Med Fam Comunidade. 2013 Abr/Jul;9(31):186-91. DOI: https://doi.org/10.5712/rbmfc9(31)867

3. Tesser CD. Prevenção quaternária para a humanização da atenção primária à saúde. Mundo Saúde. 2012;36(3):416-26.

4. Jamoulle M. Quaternary prevention: prevention as you never heard before. Belgium:WONCA International Dictionary For General/Family Practice; 2000.

5. Gomes LF, Gusso G, Jamoulle M. O ensino e a aprendizagem da prevenção quaternária. Rev Bras Med Fam Comunidade. 2015 Abr/ Jun;10(35):1-14. DOI: https://doi.org/10.5712/rbmfc10(35)1050

6. Martins C, Godycki-Cwirko M, Heleno B, Brodersen J. Prevenção quaternária: revendo o conceito. Eur J Gen Pract. 2018 Jan;24(1):106-11.

7. Brodersen J, Schwartz LM, Woloshin S. Overdiagnosis: how cancer screening can turn indolent pathology into illness. APMIS. 2014 Aug;122(8):683-9.

8. Jamoulle M. Prevenção quaternária: primeiro não causar dano. Rev Bras Med Fam Comunidade. 2015 Abr/Jun;10(35):1-3. DOI: https:// doi.org/10.5712/rbmfc10(35) 1064

9. Norman AH, Tesser CD. Prevenção quaternária na atenção primária à saúde: uma necessidade do Sistema Único de Saúde. Cad Saúde Pública. 2009 Set;25(9):2012-20.

10. Norman AH, Tesser CD. Prevenção quaternária: as bases para sua operacionalização na relação médico-paciente. Rev Bras Med Fam Comunidade. 2015 Abr/Jun;10(35):1-10. DOI: https://doi.org/10.5712/rbmfc10(35)1011

11. Moraes CF, Neiva T, Vianna LG. Prevenção em saúde na prática médica: da primária à quaternária. Rev Gest Saúde. 2015;6(2):1418-2.

12. Tesser CD. Por que é importante a prevenção quaternária na prevenção?. Rev Saúde Pública. 2017;51:116.

13. Tesser CD, Norman AH. Geoffrey Rose e o princípio da precaução: para construir a prevenção quaternária na prevenção. Interface (Botucatu). 2019;23:e180435. DOI: https://doi.org/10.1590/interface.180435

14. Harzheim E, Chueiri PS, Umpierre RN, Gonçalves MR, Siqueira ACS, D’Avila OP, et al. Telessaúde como eixo organizacional dos sistemas universais de saúde do século XXI. Rev Bras Med Fam Comunidade. 2019;14(41):1881.DOI: http://dx.doi.org/10.5712/rbmfc14(41)1881

15. Modesto AAD. Nem tudo que reluz é ouro: discutindo prevenção quaternária a partir de ditados populares. Rev Bras Med Fam Comunidade. 2019;14(41):1781. DOI: https://doi.org/10.5712/rbmfc14(41)1781

16. Tesser CD. Cuidado clínico e sobremedicalização na atenção primária à saúde. Trab Educ Saúde. 2019;17(2):e0020537. DOI: https:// doi.org/10.1590/1981-7746-sol00205

17. Barbosa AP, Ricacheneisky LF, Daudt CG. Prevenção e rastreamento de neoplasias femininas: mama e colo do útero. Acta Méd. 2018;39(2):335-45.

18. Gross DMP, Camacho ACL, Leandro R, Daher DV, Mota CP. Prevenção quaternária na gestão da atenção primária à saúde: revisão integrativa. Rev Enferm UFPE. 2016;10(4):3608-19.

19. Tesser CD, Knobel R, Andrezzo HFA, Diniz SD. Violência obstétrica e prevenção quaternária: o que é e o que fazer. Rev Bras Med Fam Comunidade. 2015;10(35):1-12. DOI: http://dx.doi.org/10.5712/rbmfc10(35)1013

20. Tesser CD, Norman AH. Differentiating clinical care from disease prevention: a prerequisite for practicing quaternary prevention. Cad Saúde Pública. 2016 Out;32(10):e00012316. DOI: https://doi.org/10.1590/0102-311X00012316

21. Norman AH, Hunter DJ, Russell AJ. Linking high-risk preventive strategy to biomedical-industry market: implications for public health. Saude Soc. 2017 Jul/Set;26(3):638-50. DOI: https://doi.org/10.1590/s0104-12902017172682

22. Mangin D, Heath I. Multimorbidade e prevenção quaternária (P4). Rev Bras Med Fam Comunidade.2015 Abr/Jun;10(35):1-5. DOI: https:// doi.org/10.5712/rbmfc10(35) 1069 
23. Modesto AAD, Lima RLB, D’Angelis AC, Augusto DK. A not-so-blue November: debating screening of prostate cancer and men's health. Interface (Botucatu). 2018;22(64):251-62.

24. Pizzanelli M, Jamoulle M. Vacinação contra o vírus do papiloma humano à luz da prevenção quaternária. Rev Bras Med Fam Comunidade. 2019;14(41):1800. DOI: https://doi.org/10.5712/rbmfc14(41)1800

25. Alber K, Kuehlein T, Schedlbauer A, Schaffer S. Medical overuse and quaternary prevention in primary care - a qualitative study with general practitioners. BMC Fam Pract. 2017;18:99. DOI: https://doi.org/10.1186/s12875-017-0667-4

26. Pausch M, Schedlbauer A, Weiss M, Kuehlein T, Hueber S. Is it really always only the others who are to blame? GP's view on medical overuse. A questionnaire study. PLoS One. 2020;15(1):e0227457. DOI: https://doi.org/10.1371/journal.pone.0227457

27. Santos JA. Autoexames mamário e testicular nos rastreamentos oncológicos: uma questão de prevenção quaternária?. Rev Bras Med Fam Comunidade. 2015 Jul/Set;10(36):1-6. DOI: https://doi.org/10.5712/rbmfc10(36)1094

28. Costa OC, Reis A. Questões epistemológicas e bioéticas da prevenção quaternária. Physis. 2012;22(4):1485-1502. DOI: https://doi. org/10.1590/S0103-73312012000400012

29. Bae JM. Implementation of quaternary prevention in the Korean healthcare system: lessons from the 2015 Middle East respiratory syndrome coronavirus outbreak in the Republic of Korea. J Prev Med Public Health. 2015 Nov;48(6):271-3.

30. Almenas M, Hidalgo CE, Pineda CA, Muñoz E, Armadillo MLR, Salvatierra E, et al. Quaternary prevention: how to do, how to teach. Rev Bras Med Fam Comunidade. 2018 Out;13(Supl 1):69-83.

31. Maldonado JMS, Marques AB, Cruz A. Telemedicine: challenges to dissemination in Brazil. Cad Saúde Pública.2016;32(Supl2):e00155615. DOI: http://dx.doi.org/10.1590/0102-311X00155615

32. Brito FG, Rodrigues AAA, Destro Filho JA. Telemedicina como instrumento de soporte en la atención primaria a la salud. Latin Am J Telehealth [Internet]. 2017; [citado 2020 Junho 09]; 4(2):155-60. Disponível em: http://cetes.medicina.ufmg.br/revista/index.php/rlat/ article/view/187/361

33. Tesser CD, D’Ávila TLC. Por que reconsiderar a indicação do rastreamento do câncer de mama?. Cad Saúde Pública.2016;32(5):e00095914 DOI: http://dx.doi.org/10.1590/0102-311X00095914

34. Treadwell J, McCartney M. Sobrediagnóstico e tratamento excessivo: médicos generalistas - é hora de uma revolução na medicina. Rev Bras Med Fam Comunidade. 2016;11(38):1-5. DOI: http://dx.doi.org/10.5712/rbmfc11(38)1400

35. Lima R. Prevenção quaternária e bioética em tempos de Covid-19. Rio de Janeiro (RJ): Sociedade Brasileira de Medicina de Família e Comunidade (SBMFC); 2020 Mai; [acesso em 202 Maio 07]. Disponível em: https://www.sbmfc.org.br/noticias/artigo-prevencao-quaternaria-e-bioetica-em-tempos-de-covid-19/

36. Widmer D, Herzig L, Jamoulle M. Prévention quaternaire: agir est-il toujours justifié en médecine de famille?. Rev Med Suisse. 2014;10(430):1052-6.

37. Pellin PP, Rosa RS. Prevenção quaternária - conceito, importância e seu papel na educação profissional. Saberes Plurais: Educação na Saúde. 2018;2(3):9-22.

38. Santos HLPC, Maciel FBM, Flores REL, Ferreira PR. Antropologia e saúde caminhos possíveis para (re)pensar a prática médica: antropologia e saúde na formação médica. REVASF [Internet]. 2019; [citado 2020 Junho 05]; 9(20):50-64. Disponível em: https://www. periodicos.univasf.edu.br/index.php/revasf/article/view/541

39. Santos HLPC, Maciel FBM. Comunicação na formação médica: algumas reflexões. REVASF [Internet]. 2020; [citado 2020 Junho 05]; 10(21):201-18. Disponível em: https://www.periodicos.univasf.edu.br/index.php/revasf/article/view/1055 